\title{
Optical Monitoring of Dip Coating: Non-Newtonian liquids
}

\author{
Alexandre F. Michels, Pedro Lovato and Flavio Horowitz \\ Instituto de Física - Universidade Federal do Rio Grande do Sul (UFRGS), \\ Campus do Vale CP15051-91501-970 Porto Alegre, RS, Brasil.
}

\begin{abstract}
Dip coated films, widely used in the coating industry, are usually measured during fabrication by capacitive methods with micrometric precision. In this work, interferometric determination of thickness evolution in real time, for the first time to our best knowledge, is applied to a class of non-Newtonian liquids with distinct viscosities. Thickness evolution during the process depends on time as predicted by a power-law model. Comparison with measured results (uncertainty of $\pm 0,007 \mu \mathrm{m}$ ) shows very good agreement after initial step of the process.
\end{abstract}

Keywords: $310.3840,310.6860,260.3160$

\section{INTRODUCTION}

Among wet bench films production processes, dip coating is widely used especially for fabricating coatings on non-planar or long-dimension objects [1]. Although dip coating requires simple equipment and small resources, its dynamics of film deposition may become complex, with different stages during the process.

In this sense, extensive theoretical studies have been devoted to describe Newtonian fluid behavior under dip coating [2, 3], as well as several empirical observations $[4,5,6,7]$. However, in these experimental studies, film thicknesses are traditionally measured by capacitive techniques with large uncertainties $( \pm 2 \mu \mathrm{m})$, which are also influenced by other parameters, such as substrate or belt thickness variations [5].

More recently, accurate optical techniques have been used to spatially measure the film profile by an interferometric technique [8,9], or for temporally monitoring physical thickness evolution during the process [10]. Comparison between experimental physical thickness evolution and theoretical results, within a precision much higher than that of the traditional capacitive method in the physical thickness determination (uncertainty of \pm $0.007 \mu \mathrm{m}$ ), are reported for Newtonian mineral oils, with constant refractive index during the dip coating process [10].

For more complex liquids, with significant refractive index variation during the process, polarimetric and interferometric techniques were combined (double optical monitoring) to determine variable refractive index and variable physical thickness values, simultaneously and in real time [11].

Application of the double-optical monitoring method to a sol-gel sulfated zirconia colloid, with variable refractive index, allowed comparison between experimental physical thickness evolution and theoretical predictions, where in the first stage, dominated by mass loss, the film was shown to be practically unaffected by evaporation and to flow as a Newtonian liquid [12].

However, in spite of the numerous theoretical-experimental papers about non-Newtonian proprieties [13, 14, $15,16,17]$, to our best knowledge still there is no optical monitoring study reporting temporal physical thickness evolution of non-Newtonian liquids during the dip coating process.

This is focused in this work, for power-law and volatile liquids (Carbopol and Carboxymethyl Cellulose-CMC), by use of the double-optical monitoring method, and aiming at a comparison between experimental results and theoretical predictions. 


\section{EXPERIMENTAL}

\section{Synthesis}

In a typical mixture of Carbopol and Carboxymethyl Cellulose (CMC), according to the Gutfinger and Tallmadge procedure [13], the Carbopol or CMC powder was first dissolved in deionized water, in distinct concentrations of 0.20 and $0.16 \mathrm{~mol} \mathrm{~L}^{-1}$. The colloidal suspension was prepared by adding powder to deionized water under magnetic stirring. Afterwards the colloidal suspension was filtrated using an FTFE membrane (Millipore with $0.2 \mathrm{~mm}$ pore size) and homogenized by ultra-sound vibration. The resultant transparent suspensions were used to coat clean silicon substrates by the dip coating process.

\section{Optical measurements}

Monitoring of the $\mathrm{CMC}$ and Carbopol film deposition properties is obtained by analysis of the reflected signal of a diode laser beam (1), at wavelength $\lambda=660 \mathrm{~nm}$. The double-optical monitoring setup, shown in Fig. 1a, can be described as follows. In the interferometric arm at normal incidence, the light transmitted by both beam splitters $(4$, 13 ) is reflected by the sample (9), in which a liquid film is flowing (Fig. 1b), and is captured by the sample detector (11). This reflected light is compared with a signal captured by the reference detector (8), and is later processed by a lock-in amplifier (not shown in Fig. la). The high acquisition rate, typically of $3.5 \mathrm{KHz}$, and the proper alignment of the optical system, leading to a small illuminated spot on the sample (diameter $<0.5 \mathrm{~mm}$ ) at $\mathrm{x}=\mathrm{x}_{0}$, are carefully kept for detailed monitoring of the dip coating process. In the polarimetric arm, at oblique incidence typically of $50-60^{\circ}$ with the substrate normal, the laser light reflected by the beam splitter (4) and mirrors $(5,7)$, after traversing the Glan-Thompson polarizer (6) with transmission axis in the plane of incidence, impinges onto the sample at a variable angle [11] and is captured by the second sample detector (10), which is connected to a computer through an A/D converter.

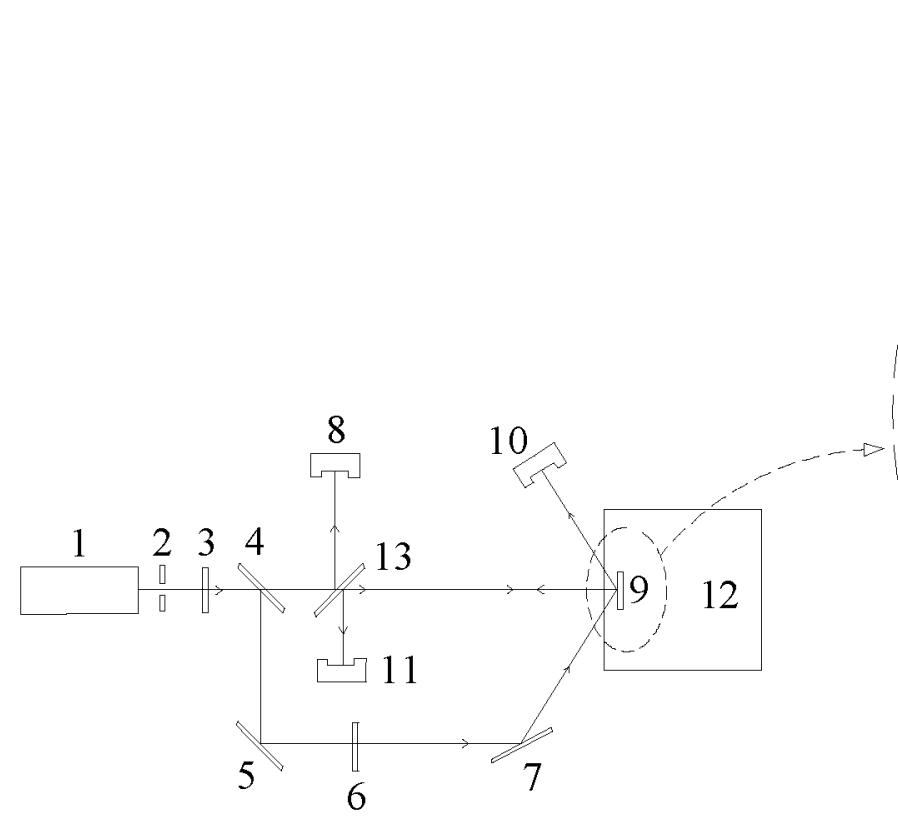

(a)

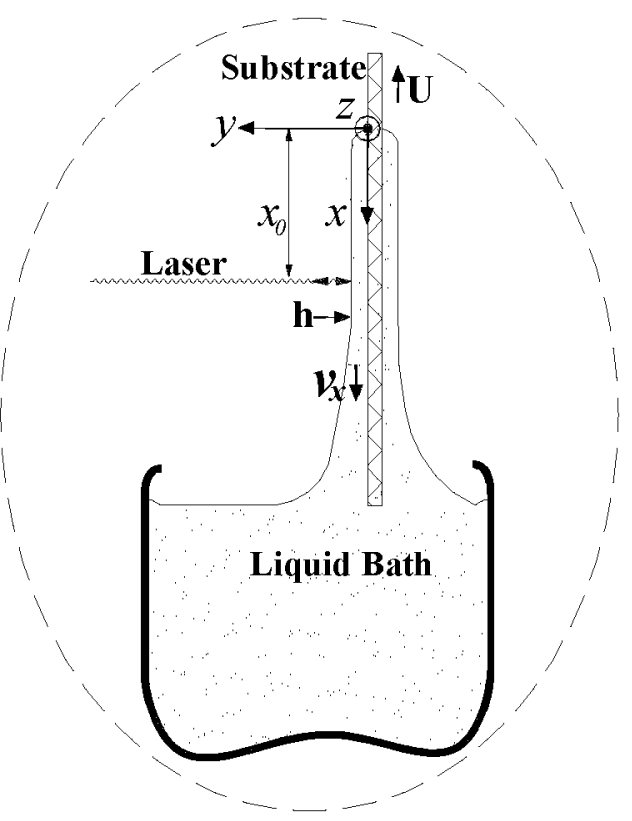

(b)

FIGURE 1. (a) Simplified scheme of the double-optical monitor, composed of laser (1), chopper (2), attenuator (3), beam splitters $(4,13)$, polarizer $(6)$, mirrors $(5,7)$, reference detector $(8)$, sample (9), sample detectors $(10,11)$ and dip coater (12). (b) Illustration of the dip coating batch process, where laser probing is shown. The spot under measurement corresponds to a fixed value in the vertical $x$-axis.

The sample and the double-optical monitoring system are vibration-isolated from the dip coater. In order to prevent contamination, as well as temperature inhomogeneities, the bath container is routinely kept inside a transparent chamber. In all experiments, the relative humidity and the temperature of the dip coater chamber are 
controlled (at $90 \%$ and $24^{\circ} \mathrm{C}$ respectively). The measuring spots, produced at low power from the attenuated $\mathrm{mW}$ laser source, do not affect the temporal pattern of the interference fringes, thus indicating that local heating does not influence film properties.

Systematic errors in optical thicknesses data were excluded by comparison with those obtained by ellipsometry. Systematic errors in refractive index data were eliminated by calibration using oil standards of known refractive indices at $25^{\circ} \mathrm{C}$, and by comparison with Abbe refractometry results at the processing temperature of $24^{\circ} \mathrm{C}$ [11].

\section{THEORETICAL}

The extremes in the interference modulated curve can be written as a function of the film optical thickness $(n h)$ as [16]:

$$
n h \cos \phi=M \lambda / 4,
$$

where $n$ is the film refractive index and $h$ its physical thickness; $\phi$ is the angle with the interface normal inside the film, $\lambda$ is the wavelength of light in vacuum, and $M$ is an integer number. In reflection, for a film with a refractive index larger than that of the substrate, Equation (1) applies to interference minima for $M$ even and to interference maxima for $M$ odd.

Physical thickness evolution of a liquid film on a substrate with infinite length in the dip coating continuous process can be described, under steady-state flow, by the Gutfinger and Tallmadge equation [13]:

$$
h(x, t)=\left(\frac{K}{\rho g}\right)^{\frac{1}{s+1}}\left(\frac{x}{t}\right)^{\frac{s}{s+1}},
$$

where $x / t$ corresponds to the withdrawing speed $U$ for an observer in the laboratory reference system, where $s$ is the power index and $K$ is the apparent non-Newtonian viscosity, $\rho$ is the liquid density and $g$ the gravity force.

\section{RESULTS AND DISCUSSION}

By use of the Double Optical Monitoring Method [11], experimental results were obtained for two volatile non-Newtonian liquids (Carbopol and CMC) with distinct viscosities.

Figure 2 shows interferograms obtained from the temporal evolution of Carbopol, where each interval between successive extremes corresponds to an optical thickness (physical thickness multiplied by refractive index) variation of $\lambda / 4$.

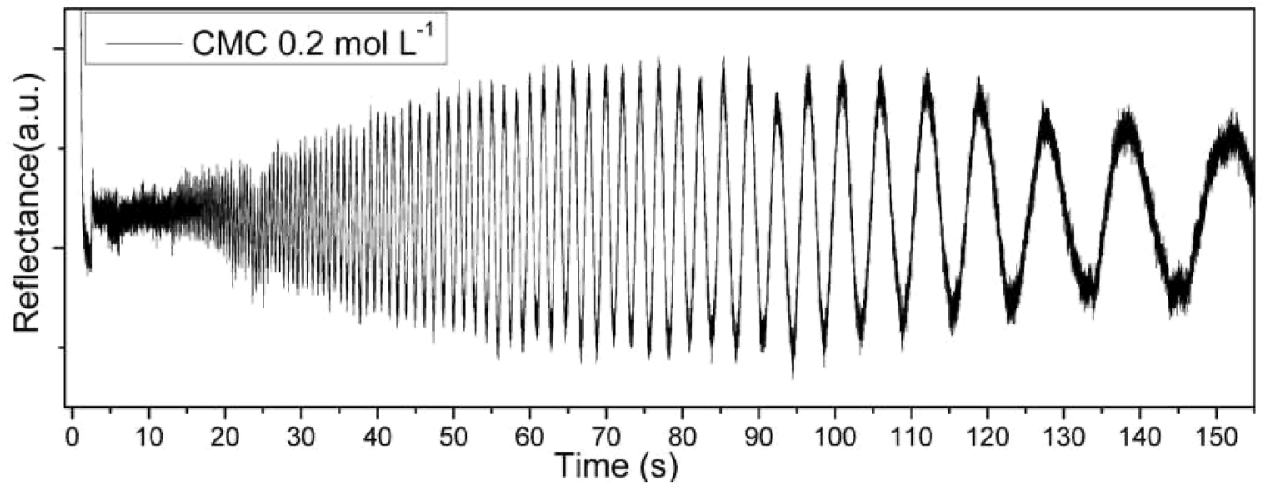

FIGURE 2. Reflectance temporal evolution for Carboxymethyl Cellulose (CMC), during dip coating.

Through these $\lambda / 4$ step intervals, and by direct polarimetric measurement of the refractive index in real time, information about physical thickness evolution was achieved during the process, as shown in figure 3 . 


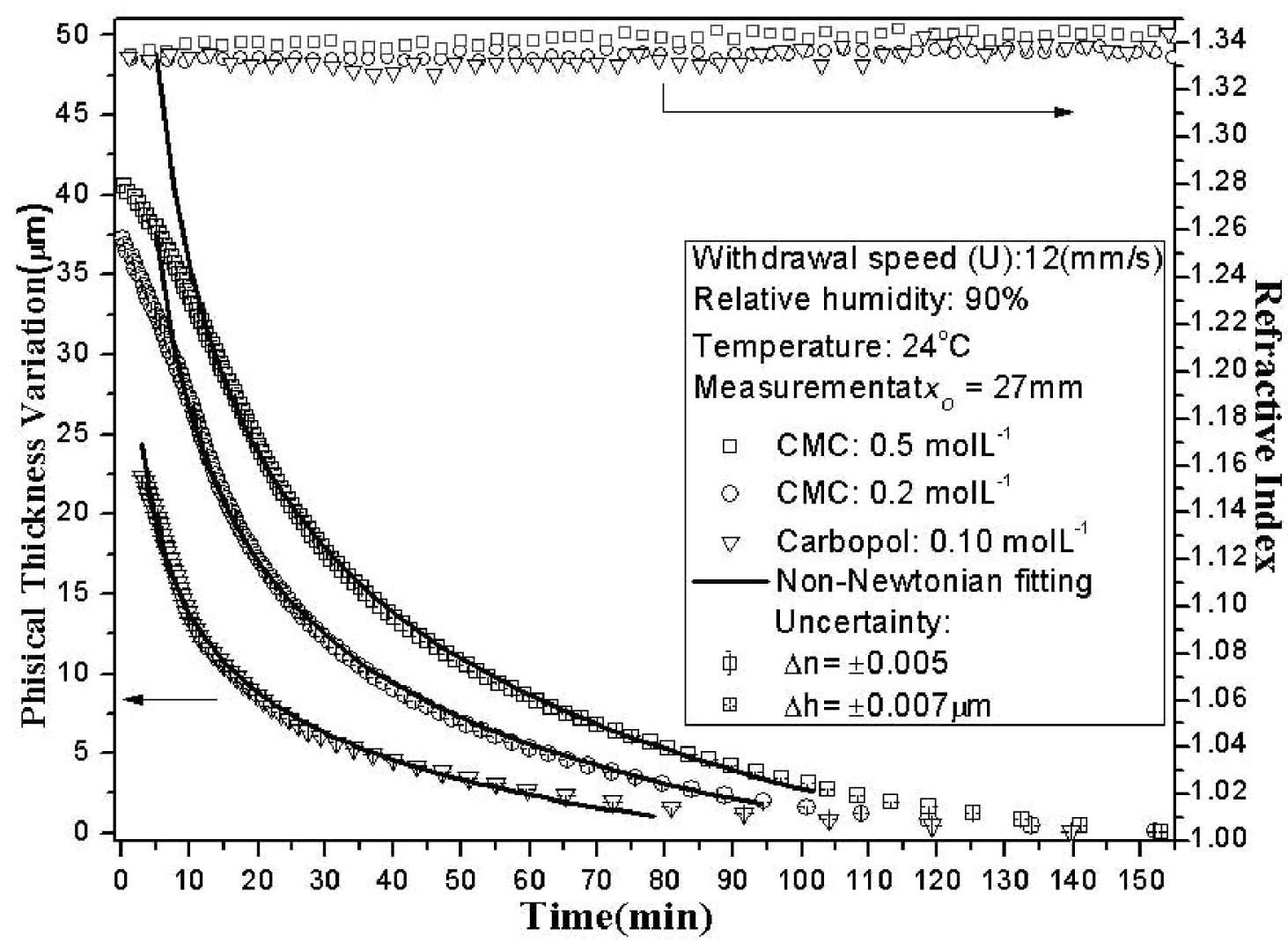

FIGURE 3.Temporal evolution of physical thickness and refractive index during dip coating of Carbopol and Carboxymethyl Cellulose (CMC) at distinct concentrations. Although experimental points were obtained at each $\lambda / 4$ variation, only data at each $\lambda$ variation are shown for better visualization. Modeling results are shown in solid curves. Displayed uncertainties are specified at the in set.

In this figure, we note that as the CMC concentration increases, so does the mass loss in the same time interval. This does not imply in a smaller optical thickness value, which would contradict equation (2), since the initial thickness is larger for a higher viscosity.

Now we focus on the comparison between experimental results and the theoretical power-law predictions shown as a solid line in Figure 3. The agreement attained, after the very initial moments of the process, indicates that the experimental data, within the uncertainty of $\pm 7 \mathrm{~nm}$, agree well with the values obtained from Non-Newtonian power-law relation (2). The theoretical fitting parameters, $K$ and $s$, are compatible with previous reports by Gutfinger and Tallmadge involving Carbopol [15].

\section{CONCLUDING REMARKS}

First double-optical analysis during batch dip coating of non-Newtonian fluids was presented. Quantitative description of temporal thickness variation was then achieved since the beginning of the process at an acquisition rate of $3.5 \mathrm{KHz}$.

After the initial few seconds, the experimental data agreed very well with the dependence predicted by the power-law model. The precision of the optical method in thickness variation during the dip coating process is two orders of magnitude better than that attained by traditional capacitive measurements. As a consequence, the optical method allows characterization of liquid layer thicknesses with nanometric precision, thus opening new perspectives for further characterization and understanding of the process temporal dynamics of Non-Newtonian liquids.

\section{ACKNOWLEDGMENTS}

We are grateful to support from CNPq - Conselho Nacional de Desenvolvimento Científico e Tecnológico, in collaborative project with researcher Fabian Fagotti at Empresa Brasileira de Compressores - EMBRACO 


\section{REFERENCES}

1. 1. L.E. Scriven, Materials Research Society, 121, 717 (1988).

2. L.D. Landau and B.G. Levich, Acta Physicochim. 17, 42 (1942).

3. .G. Levich, Physicochemical Hydrodynamics (Prentice-Hall, New Jersey, 1962, chap. 12.

4. D. A. White and J. A. Tallmadge, Chem. Eng. Sci. 20, 33-37 (1965).

5. P. Groenveld, Chem. Eng. Sci. 25, 33 (1970).

6. R.P. Spiers, C.V. Subabaraman and W.L. Wilkinson, Chem. Eng. Sci, 29, 389 (1973).

7. P. R. Schunk, A. J. Hurd and C. J. Brinker, Chapter 13 in Liquid Film Coating, S. F. Kistler and P. M. Schweizer eds., London, (1997)

8. F. Nishida, J.M. McKiernan, B. Dunn, J.I. Zink, C.J. Brinker, A.J. Hurd, J. Am. Ceram. Soc., 78, 1640 (1995).

9. D. Qu, E. Rance', and S. Garoff, Phys. Fluids 14, 1154-1156 (2002)_.

10. A. F. Michels, T. Menegotto, and F. Horowitz, Appl. Opt. 43, 820-823 (2004).

11. A.F. Michels, T. Menegotto, H.P. Grieneisen, M.B. Susin, Applied Optics Special Issue on Optical Interference Coatings, Appl. Opt. 45, 7, 1491 (2006).

12 A.F. Michels, T. Menegotto, C.V. Santilli, F. Horowitz, J. Non-Cryst. Solids, 352, 5362-5367(2006)

13. C. Gutfinger and J.A. Tallmadge, A.I.C.h.E. Joumal 11, N 3, 403 (1965).

14. R.P. Spiers, C.V. Subabaraman and W.L. Wilkinson Chem. Eng. Sci, 30, 379-385 (1975).

15. J. A. Tallmadge, AIChE. J. 16, 925-930 (1969)

16. M. Born, E. Wolf, Principles of optics: electromagnetic theory of propagation, interference and diffraction of light. 6. ed. Cambridge University Press, 1997, pp. 51-64. 
Copyright of AIP Conference Proceedings is the property of American Institute of Physics and its content may not be copied or emailed to multiple sites or posted to a listserv without the copyright holder's express written permission. However, users may print, download, or email articles for individual use. 